\title{
COMBINED SPINAL EPIDURAL ANESTHESIA FOR ANTERIOR APPROACH TOTAL HIP ARTHROPLASTY
}

YENIOCAK T., CANBOLAT N. Baltalimani Metin Sabanci Bone and Joint Diseases Education and Research Hospital ,Istanbul, TURKEY

Department of Anaesthesiology

\section{Background and Aims:}

Anterior approach (AA) is a new approach for total hip arthroplasty (HA). AA has the advantages of minimum soft tissue damage, improved early recovery and decreased rates of dislocation compared other approaches. Neuroaxial block is known as the gold standard for HA perioperatively and postoperative pain management. The aim of the study is to report a case of combined hemi spinal epidural anesthesia (CSEA) for AAHA.

\section{Methods:}

A 58-year old male was presented for AAHA. He has severe asthma with the following spirometry results: FVC: \%42, FEV1: \%35 and FEV1/FVC: \%55. Also liver function tests were idiopatically high; ALT: 198 and AST: 163. The patient is considered as morbidly obese ; BMI :42. General anesthesia was not suggested on account of hepatotoxicity and predicted extubation difficulties. On arrival to the operation room, standart monitoring was applied by electrocardiography, pulse oximetry and invasive arterial blood pressure monitoring. The patient was administered $8 \mathrm{ml} / \mathrm{kg}$ body weight of Isolyte solution by 18-gauge cannula placed in a forearm vein. The patient was placed in the lateral decubitis position with the non operation side in the upper position. We performed hemi spinal anesthesia with $2 \mathrm{ml} \% 0.5$ bupivacaine heavy. Epidural catheter was administered for postoperative pain management.

\section{Results:}

No heamodynamic or systemic changes occurred perioperatively. Operation ended uneventfully. The patient was transferred to his room with a patient control analgesia (PCA) device via epidural catheter.

\section{Conclusions:}

We are not aware of literature published on the anesthetic technique applied with the AAHA. Physicians may be concerned about the application of neuroaxial blocks because the procedure requires the patient to be in the trendelenburg position. However, the patient is placed in the trendelenburg position only during the last phase of the operation, during which the neuroaxial block is already set. Completed spinal block or hypotension caused by positioning risk is extremely low in the last phase of the operation. Further prospective randomized controlled trials for the AAHA technique should be performed on an increased number of cases to evaluate the results 